The clinicians should be aware of this complication with high mortality especially in pediatric patients. In CD HLH is probably developing as combination of several factors: primary disease, immunomodulation and infection (primary or reactivation).

\section{ANOTHER FACE OF EPSTEIN-BARR VIRAL INFECTION: ACUTE ACALCULOUS CHOLECYSTITIS AND SEVERE LIVER LESION IN PREVIOUSLY HEALTHY FIVE-YEAR-OLD GIRL WITH PRIMOINFECTION}

Tena Trbojević, Iva Škorić, Paula Slatina, Frane Marleku, Orjena Žaja. Department of Pediatrics, Sestre milosrdnice University Hospital Center, Zagreb, Croatia

10.1136/archdischild-2021-europaediatrics.257

Acute acalculous cholecystitis (AAC) is a rare disease, particularly in children. It is mostly observed in critically ill or postsurgical patients due to dehydration, increased bile viscosity, decreased oral intake and gallbladder dysmotility. Most AACs in children are caused by infectious agents and are characterized by favorable clinical course and conservative treatment. Also, AAC is an atypical and rare complication of EpsteinBarr virus (EBV) infection. The occurrence of AAC has been described in some chronic disorders and systemic autoimmune diseases. The clinical and laboratory presentation of AAC is variable. The diagnosis is based on radiological criteria. Abdominal ultrasound typically shows distended gallbladder with thickened wall, pericholecystic fluid or edema and absence of gallstones or dilatation/obstruction of bile ducts.

We present a girl with AAC and acute severe liver lesion due to the EBV primoinfection.

Previously healthy 5-year-old-girl was admitted to the Pediatric Department (April 2020) due to jaundice, severe liver lesion and cholestasis. She was inapetent for a week; had pruritus, pale and yellowish skin, occasional abdominal pain and one hypocholic stool. No fever, nausea or vomitting was reported. At admittance, icterus, an enlarged liver ( $3 \mathrm{~cm}$ in the medioclavicular line) and a negative Murphy's sign were noticed. She was afebrile, HR 132/min, BP 80/50 $\mathrm{mmHg}$; body height $116.3 \mathrm{~cm}$ (88th centile), BMI $18 \mathrm{~kg} /$ m2 (93rd centile). She had no lymphadenopathy or pharyngitis.

Laboratory tests revealed severe hepatitis and conjugated hyperbilirubinemia (AST 1908 U/L, ALT 3222 U/L, GGT 51 $\mathrm{U} / \mathrm{L}$, bilirubin total $83.6 \mu \mathrm{mol} / \mathrm{L}$, conjugated $68.5 \mu \mathrm{mol} / \mathrm{L}$, total serum bile acids $61.2 \mu \mathrm{mol} / \mathrm{L}$ ) with preserved synthetic and metabolic liver function and normal values of inflammatory markers. Ultrasonography revealed mild hepatosplenomegaly, hyperechogenic liver parenchyma, normal bile ducts and slightly dilatated gallbladder with thickened and layered wall, significant pericholecystic edema and the absence of gallstones. The following assessment found no elements of malignancy, autoimmune etiology, chronic primary and secondary liver diseases, dyslipidemia, bacterial or parasitic infection. Serological analysis confirmed EBV primoinfection and significant viremia (28 000 copies $/ \mathrm{ml}$ at PCR test). Conservative treatment was followed by spontaneous clinical, laboratory and radiological regression.

We presented a rare hepato-biliary manifestation of EBV infection in children. Our aim was to highlight the importance of recognizing $\mathrm{AAC}$ as a differential diagnosis in children with abdominal pain and/or acute cholestasis. Radiological evaluation and monitoring as well as collaboration of gastroenterologists and radiologists are essential in management of these patients.

\section{A RARE CASE OF ACUTE PANCREATITIS AND REACTIVE ARTHRITIS IN ROTAVIRUS GASTROENTERITIS}

Matea Crnković Ćuk* ${ }^{*}$ Barbara Perše, Mandica Vidović, Orjena Žaja. Clinical Hospital Centre Sestre milosrdnice

10.1136/archdischild-2021-europaediatrics.258

Pancreatitis in children is an acute inflammation of the pancreas that manifests itself with abdominal pain localized in the epigastrium and elevated levels of enzymes secreted by the pancreas and is a significant cause of mortality and morbidity. Hyperamylasemia and hyper lipasemia occur relatively frequently in acute gastroenteritis, but pancreatitis itself is relatively rare. Reactive arthritis can also often be associated with acute viral infections although it is extremely rarely described with rotavirus infection.

We present a rare case of pancreatitis and reactive arthritis associated with rotavirus gastroenteritis.

A 5-year-old, 9-month-old girl presented with severe epigastric pain, vomiting, and sub febrile condition that lasted for 24 hours.

Family and personal anamnesis were without peculiarities. At admission, severe pain in the epigastrium, sub febrile and increased peristalsis without other deviations were noted. Leukocytosis $(19.3 \times 10 \wedge 9,86 \%$ of segmented leukocytes), elevated CRP (19.9 mg/L) and markedly elevated lipase (4025 U/ $\mathrm{L})$, serum amylase present in laboratory findings

$\mathrm{U} / \mathrm{L}$ ) and urine (13439 U/L). Rotavirus antigen was isolated in stool by negative other microbiological analysis. No pathology of the hepatobiliary tree, with minimal changes of the pancreas, in terms of hypo echogenicity without effusion was detected by ultrasound. She did not develop cystic lesions.

Upon admission, oral intake was discontinued and parenteral hydration was initiated.

Intensive monitoring shows a favourable trend of rapid decline in amylase and lipase values. After 72 hours, oral administration was started with gradual discontinuation of the intravenous infusion, which was well tolerated. The diet continued with the accelerated introduction of protein and fat without relapse. She was febrile for 3 days with rapid clinical recovery. On the seventh day of the stay, complains of pain and swelling of the dorsum of the left foot with a renewed increase in inflammatory parameters (CRP $27.4 \mathrm{mg} / \mathrm{L}$, SE 52 $\mathrm{mm} / 3.6 \mathrm{hp}$ ). Through clinical evaluation and ultrasound findings, arthritis was suspected (in the projection of the navicular and cuneiform bone, synovitis and enhanced power - doppler signal). Given the rapid clinical recovery over the next 2 days, without the need for further diagnostic or therapeutic interventions, it was concluded that this was transient reactive arthritis.

Rotavirus is a common cause of severe diarrhoea in children worldwide and it is a significant cause of morbidity in developed countries and mortality in developing countries. Although extremely rare, associated pancreatitis is a serious and potentially fatal extra intestinal complication and further 
emphasizes the importance of vaccination, especially in infants and young children.

\section{CLINICAL, ECONOMIC AND HUMANISTIC IMPACT OF SHORT BOWEL SYNDROME - INTESTINAL FAILURE IN PORTUGUESE PAEDIATRIC PATIENTS (PARENTERAL STUDY)}

Raúl Barbosa da Silva, Sara Nóbrega, Paula Guerra, Ricardo Ferreira, Diogo Ramos*, Valeska Andreozzi, Marisa D Santos. Exigo Consultores

\subsection{6/archdischild-2021-europaediatrics.259}

Short Bowel Syndrome - Intestinal Failure (SBS-IF) is a rare, chronic, and debilitating disease, requiring patient-tailored complex management and comprehensive care. Parenteral nutrition (PN) remains the standard-of-care due to its life-sustaining nature. This study aimed to assess the clinical, economic and humanistic impact of paediatric SBS-IF in Portugal.

This was a retrospective cohort study, with a cross-sectional component for the quality of life (QoL) evaluation, including paediatric SBS-IF patients

( $\geq 1$-18 years) with stable PN over at least six months. Data collection included patient chart review over a 12-month period and patient/caregiver self-report and PedsQL ${ }^{\mathrm{TM}}$ questionnaires with 6- and 1-month recall periods, respectively. Main endpoints included clinical and PN characterization, healthcare resource utilization (HRU), direct medical and non-medical costs, and patient QoL. Unit costs were obtained from national databases/decrees, retailers, and patient/caregiver. Costs were standardized using the 2019 consumer price index and annualized assuming constant use of resources.

A total of 20 patients were included with a mean age (SD) of $7.5(5.0)$ years, $50.0 \%$ female, and a mean time since diagnosis of 6.6 (4.2) years. The three leading causes of SBS-IF were volvulus (40.0\%), intestinal atresia $(35.0 \%)$, and necrotizing enterocolitis (10.0\%). PN was administered for a mean of 6.6 days/week, in $90.0 \%$ of cases at home for a mean of 10.8 months/year. Nevertheless, $60.0 \%$ had PN administered at least once in an inpatient setting for a mean of 1.8 months/ year. HRU was high, including a mean annual frequency of 10.2 (5.3) medical visits, 29.8 (85.3) visits with other healthcare professionals (including nurse, dietitian, psychologist),

3.0 (2.5) emergency visits, and 2.0 (1.5) hospitalisations. A total of 40 hospitalizations were reported, with a mean annual length of stay of 29.4

(32.3) days, of which $85.0 \%$ due to catheter-related complications, including septicaemia, local central venous catheter infection, and mechanical complications. Mean annual direct costs $(95 \% \mathrm{CI})$ per patient amounted to $74,734.5 €(74,614.8$ $74,854.4)$, with PN and hospitalization as the main cost drivers accounting for $57.3 \%$ and $21.0 \%$ of overall costs, respectively.

Patient QoL assessment showed a PedsQL ${ }^{\mathrm{TM}}$ mean total score slightly below the Portuguese norm (73.3 vs. 75.6 ), but with a notorious deterioration in the school functioning domain (57.5 vs. 72.6).

Paediatric SBS-IF management is characterized by a substantial therapeutic burden and HRU, translating into high direct costs and QoL deterioration, mainly in school performance. There is a clear unmet need for therapeutic alternatives that lower SBS-IF burden. This research was funded by Takeda.

\section{0 CLUSTER BREASTFEEDING SYNDROME IN INFANTS}

Olga Gumeniuk*, Gumeniuk Olga, Nikolaeva Nataliia, Chernenkov Yuriy. Saratov State Medical University

10.1136/archdischild-2021-europaediatrics. 260

Cluster breastfeeding syndrome («cluster feedings», «bunch feedings") is when baby has several feedings close together during a certain period of time, in some cases during the day. The purpose of this study is to examine the frequency and to identify the reasons for the cluster breastfeeding syndrome in lactating women.

Questioning was conducted with 223 lactating women aged 19-44 years.

Questioning including questions regarding the use of the delivery methods and the lactation period. Psychological testing of lactating women was also conducted. The anxiety level was evaluated by Hamilton Anxiety Rating Scale

(HAM-A) (score of 14 points is borderline). Lactating women were divided into two groups: without Cluster Breastfeeding syndrome (group I) and with Cluster Breastfeeding syndrome (control group). Statistical analysis was performed using Microsoft Excel 2007, SPSS Statistics v 24.0.0.0. Spearman's correlation coefficient ( $r$ ) and Pearson's correlation coefficient (rxy) were calculated. Data was compared using chisquare test and $\mathrm{P} \leq 0.05$ was regarded as statistically significant.

Among the women surveyed, women with one child prevailed (63\%). The average duration of the lactation period was $11.1 \pm 5$ months. The average time the baby was at the breast was $19 \pm 4.1$ minutes. Syndrome of prolonged, continuous feeding was noted in $5 \%$ of cases when the baby was at the chest continuously for a day, with short breaks at night sleep. In this group, in women (90\%), labor was performed by Caesarean section. In the group I (cluster breastfeeding syndrome group), the average age of women was $35.8 \pm$

5.5 years, in the control group $25.0 \pm 4.6$ ( $\mathrm{p}<0.001$ ). In group I, the average score on the Hamilton scale was $28.4 \pm$ 6.5 (level of symptomatic anxiety), in the control group $12.9 \pm 9.7(p=0.0003)$. All baby in the group I have been gaining enough weight and producing sufficient dirty and wet diapers. Correlation analysis revealed a direct strong correlation between the presence of cluster breastfeeding syndrome in a child born by Caesarean section $(r x y=0.97)$ from mothers who gave birth over the age of 35 and have a level of symptomatic anxiety ( $\mathrm{r}=1)$.

Cluster breastfeeding syndrome (cluster feedings, bunch feedings) occurs in 5\% of cases and is associated with the late birth of the first child, high anxiety of the mother and the birth of a child by Caesarean section.

\section{INFANT HEMATEMESIS: A CHALLENGING DIAGNOSIS, POSTNATAL CMV INFECTION OR NON IGE-MEDIATED COW'S MILK PROTEIN ALLERGY? A TWO CASE REPORT COMPARISON}

Mara Fumagalli*, Laura Gianolio, Vania Giacomet, Gian Vincenzo Zuccotti. Department of Pediatric Infectious Diseases, Sacco Hospital, University of Milan, Italy

10.1136/archdischild-2021-europaediatrics.261

Introduction While severe manifestations of congenital Cytomegalovirus (CMV) transmission are widely investigated, little 